Lung cancer is the leading cause of cancer-related death worldwide. Although treatment methods such as surgery, radiotherapy and/or chemotherapy have improved, prognosis remains unsatisfactory, and developing new therapeutic strategies is still an urgent matter. Immunotherapy is a novel therapeutic approach wherein activated immune cells can specifically kill tumour cells. Several lung cancer vaccines have demonstrated prolonged survival time in phase II and III trials, and several clinical trials are under investigation. However, many clinical trials involving cancer vaccination with defined tumour antigens have shown this method to work only in a small number of patients. Cancer immunotherapy is not completely effective in eradicating tumour cells because they evade host immune control.

Key words: non-small cell lung cancer, immunotherapy, vaccines.

Contemp Oncol (Pozn) 2014; 18 (2): 77-84 DOI: $10.5114 /$ wo.2014.42724

\section{Actual status of therapeutic vaccination in non-small cell lung cancer}

Katarzyna Szyszka-Barth¹, Katarzyna Ramlau², Joanna Goździk-Spychalska³, Łukasz Spychalski ${ }^{1}$, Maciej Bryl ${ }^{1}$, Iwona Gołda-Gocka' ${ }^{1}$, Anna Kopczyńska ${ }^{4}$, Aleksander Barinow-Wojewódzki ${ }^{5}$, Rodryg Ramlau ${ }^{1,6}$

${ }^{1}$ Department of Clinical Oncology with the Subdepartment of Diurnal Chemotherapy, Wielkopolska Center of Pulmonology and Thoracosurgery of Eugenia and Janusz Zeyland, Poznan, Poland

${ }^{2}$ Clinic Hospital, Poznan University of Medical Sciences, Poland

${ }^{3}$ Department of Pulmonology, Allergology and Lung Oncology, Poznan University of Medical Sciences, Poland

${ }^{4}$ Department of Chemotherapy, Poznan University of Medical Sciences, Poland

${ }^{5}$ Adult Pulmonary-Rehabilitation Department, Wielkopolska Center of Pulmonology and Thoracosurgery of Eugenia and Janusz Zeyland, Poznan, Poland

${ }^{6}$ Thoracosurgery Clinic, Poznan University of Medical Sciences, Poland

\section{Introduction}

Lung cancer is the most common cause of cancer-related death worldwide. In spite of the improving results of treatment utilizing surgery, radiation therapy and/or chemotherapy, prognosis remains unsatisfactory, and new therapeutic strategies are urgently needed. The aim of this paper is to discuss the identification of tumour antigens in lung cancer, tumour immune escape mechanisms, the history of immunotherapy in NSCLC and clinical trials devoted to its use [1].

\section{Definition and history of immunotherapy}

Immunotherapy began to be employed for neoplastic diseases at the end of the $19^{\text {th }}$ century. In 1890, a New York surgeon named William Coley observed the spontaneous regression of a facial sarcoma in a patient, in whom bacterial infection occurred postoperatively [2]. Further research into this phenomenon led to the development of the substance known as Coley's vaccine - a mixture obtained from Streptococcus pyogenes and Serratia marcescens bacterial cultures. The first patient in whom Coley's toxin was employed was a 16-year-old boy with advanced neoplasm located in the abdominal cavity. Daily injections of the vaccine directly into the tumour caused the patient to exhibit symptoms of infection, including fever and shivering, but the treatment, which lasted for several months, resulted in significant regression of the tumour [3]. Notwithstanding, the substance responsible for the antineoplastic effect induced by bacterial toxins was not identified for the next 100 years. It then turned out to be a protein produced by macrophages of the recipient, which became known as tumour necrosis factor $\alpha$ (TNF- $\alpha)$ [4]. Since that time, numerous clinical studies have been conducted, employing various strategies of stimulating the immune system to combat the neoplasm. One example consisted in attempts to use Corynebacterium parvum bacteria, one of the stronger non-specific immune system stimulators. In 1908, Albert Calmette and Camille Guérin began working with an attenuated form of bovine bacillus (Myobacterium bovis), the Bacillus Calmette-Guérin strain (BCG), in order to develop a vaccine against tuberculosis. Concurrently, lower incidence of neoplastic diseases was observed among patients with active tuberculosis. These observations resulted in the 
development of a vaccine using the BCG strain as a form of immunotherapy for neoplastic diseases; it still remains an accepted form of treating bladder cancer [5]. The modern era of immunotherapy began with the research conducted by Steven Rosenberg, who was the first to employ adoptive $T$ lymphocytes and recombinant interleukin 2 in the treatment of advanced melanoma [6].

Passive immunotherapy is defined as administration of an immunologically active agent produced outside the host's body. Examples of passive immunotherapy include the administration of monoclonal antibodies or treatment with adoptive $T$ lymphocytes. Active immunotherapy focuses on stimulation of the host's immune system to eradicate cancer, either by means of vaccines containing tumour antigens combined with adjuvants (active specific immunotherapy) or by using non-specific immunomodulators [7]

\section{Antineoplastic immunological response and the mechanisms by which tumour cells evade immune system control}

Immune cellular response is initiated by the uptake of tumour-associated antigens (TAAs) by antigen-presenting cells (APCS), among which the most crucial role is played by dendritic cells [7]. Antigens are internalised as short peptide sequences and are presented on the extracellular surface of APCs in combination with major histocompatibility complex (MHC) class II molecules. Dendritic cells, together with the peptide antigens on the surface, mature within lymph nodes, where they come into contact with "virgin" $\mathrm{T}$ lymphocytes. These conditions are essential in order to initiate the activation of specific effector $T$ lymphocytes: cytotoxic CD8+ T-cells and helper CD4+ T-cells. It requires complex interactions between the TAA-MHC complex on the APC surface and the receptor on the "naive/virgin" T lymphocyte, as well as a co-stimulating signal, consisting of interactions between B7.1 (CD80) and B7.2 (CD86) proteins on antigen-presenting cells and CD28 proteins on the surface of T lymphocytes [8]. The activation of helper CD4+ lymphocytes leads to the secretion of a number of cytokines (including, among others, IL-2, IL-12 and IFN-gamma), which activate CD8+ lymphocytes directly or through macrophage mediation. The presence of the cytokines (secreted by the stimulated immune system cells) increases the activity of natural killer (NK) cells, enhances the phagocytic capability of macrophages, improves the synthesis of specific antibodies by $\mathrm{B}$ lymphocytes and directly eradicates cancer cells or inhibits their proliferation [9]. Despite clear and confirmed evidence for the existence of immunological response targeted against neoplastic cells, the tumours usually continue to grow. This phenomenon became the reason for the search for the mechanisms in which tumour cells evade immune system control, and it explained the failure of earlier research into therapeutic cancer vaccines [10-12]. The evasion mechanisms include, among others, the immunomodulation of tumour cells by the selection of antigen-loss variants, which consists of the tumour cell population losing its ability to express antigens inducing antitumoral response [13]. Another way of avoiding immune response is the tolerance mechanism, in which the immune system is not activated against the tumour-cell antigens, because the antigens are recognized as self. The state of immunological tolerance is related to the ability of naive/virgin $T$ lymphocytes to ignore self antigens and to the active elimination of autoreactive $T$ cells. Most TAAs are non-mutated antigens present on normal tissue, which means that the host must have developed tolerance to these antigens. In at least two mouse models, tolerance to the antigen present in the administered vaccine was observed, which suggested that more frequent use of immunotherapy may lead to immune tolerance induction being increasingly visible [14]. Moreover, tumour cells actively change their phenotype. One of the reasons for this is downregulation, i.e. reducing the number of MHC molecules on the surface of tumour cells. In theory, this should disrupt the presentation of tumour-associated antigens to virgin cytotoxic CD8+ T lymphocytes. Also significant is the secretion of the so-called immunoinhibitors, which include the cytokine called transforming growth factor beta (TGF- $\beta$ ), which inhibits various functions of lymphocytes and macrophages and is secreted by many types of neoplastic tumours. It has been demonstrated that TGF inhibits the proliferation of $T$ lymphocytes, the maturation of cytotoxic $T$ lymphocytes (CTLS) and the activation of macrophages. Interleukin 10, secreted by tumour cells, operates in a similar fashion, inhibiting the proliferation of T cells $[15,16]$. Another way in which tumours evade immune system surveillance is by inducing the apoptosis of T lymphocytes with Fas proteins on their cell membranes, attacking the tumour cells $[17,18]$.

\section{Therapeutic vaccines in the treatment of non- small-cell lung carcinoma patients}

Phase 2 and 3 clinical trials assess vaccines employed at different stages of cancer progression. With regard to complementary treatment after radical surgical procedures (after complementary chemotherapy or without it), phase 3 trials assess the MAGE-A3 vaccine (advancement stage IB-IIIA), and phase 2 trials assess the PRAME vaccine (stage IA-IIIA). The phase 3 trial assessing the efficacy of the Stimuvax vaccine (stage III patients after the completion of chemoradiotherapy) ended unsuccessfully. The phase 3 trial assessing the efficacy of the Lucanix vaccine in IIIA (T3N2)-IV stage patients after the completion of chemotherapy or chemoradiotherapy was also terminated. CimaVax and TG4010 vaccines are useful for IIIB/IV stage patients in combination with chemotherapy (TG4010) or after the completion of cytostatic treatment (CimaVax).

\section{MAGE-A3 vaccine}

MAGE-A3 is a gene which encodes the MAGE-A3 protein (Melanoma-Associated Antigen A3). The gene is silent in all human tissues except for the testes. Expression in testicular cells does not, however, result in the destruction of these cells by immunotherapy, because they are unable to present antigens to the immune system. MAGE antigens were first identified in malignant melanoma cells, based on the ability of these cells to stimulate autologous 
cytotoxic T lymphocytes [7]. MAGE-A3 antigen expression can be observed in many malignant tumours, including non-small cell lung carcinoma (NSCLC), where the expression oscillates within the range 35-50\%, depending on the stage of the tumour's clinical advancement (approximately $35 \%$ in stage IB and II, and approximately $50 \%$ in stage III) [19-22]. Antigen expression is an independent unfavourable prognostic factor, and its indexes are higher in squamous cell carcinoma in comparison with adenocarcinoma [23]. MAGE contains epitopes that can be presented by major histocompatibility complex class I and II molecules. This explains the ability to simultaneously activate both CD8+ and CD4+ T lymphocytes. MAGE-A3 immunotherapy is, therefore, an antigen-specific form of immunotherapy, responsible for the induction of a strong immune response, both humoral and cellular [24]. Immune response induction as a result of MAGE vaccination in patients with lung cancer was first observed and identified in 2004. Based on this finding, a clinical study was designed which included 17 patients after surgery, with no discernible tumour foci detected by imaging examinations. Nine of the patients were receiving $300 \mu \mathrm{g}$ of MAGE protein, while 8 were receiving MAGE in combination with ASO2B adjuvant [25]. In the first cohort, only 1 patient exhibited MAGE-A3-specific response of CD4+ T cells, while as many as 4 patients from the second cohort developed a CD4+ response against the MAGE-A3DP4 protein. This became the basis for starting an international randomized phase 2 trial, which encompassed 182 patients (in stages IB and II) after complete resection of NSCLC whose cells exhibited MAGE-A3 expression. The patients were randomly allocated to the arm receiving the MAGE vaccine or to the arm receiving placebo in a 2:1 ratio. The study arm with the vaccine exhibited a trend toward a longer disease-free interval, as compared with the placebo arm $(H R=0.74$; $p=0.107$ ), with similar non-significant improvements in terms of disease-free survival (DFS) and overall survival (OS) [26]. The disease recurred in $30.6 \%$ patients in the vaccine arm and in $43.4 \%$ patients in the placebo arm. During the study, only three vaccine-related adverse effects were reported (CTCAE grades 3 and 4) [27]. Clinical efficacy of the MAGE-A3 vaccine was nearly two-times higher in the group of patients with gene signatures associated with high risk of recurrence $[28,29]$. The results obtained during phase 2 became the foundation for designing a randomized phase 3 trial conducted in 33 countries; recruitment for this study was completed by the end of 2011. The MAGRIT study (MAGE-A3 as Adjuvant Non-Small Cell Lung Cancer Immunotherapy) randomized 2270 patients after radical surgical procedures for NSCLC (stages IB-IIIA), whose tumours exhibited MAGE expression. The patients were randomly allocated to the arm receiving the MAGE vaccine or to the arm receiving placebo in a ratio of $2: 1$. The immunotherapy involved 13 intramuscular injections within a period of 27 months ( 5 injections every 3 weeks with subsequent supportive treatment -1 injection every 3 months) [7]. The study protocol stipulated provision of immunotherapy to patients receiving adjuvant chemotherapy based on platinum derivatives and to patients not receiving complementary chemotherapy.
Prospectively, the gene signature was also assessed, but it was not taken under consideration as a stratification factor during randomisation [27].

The immunoadjuvant used in this study was AS15 in liposomal form. It is a strong immunostimulant, capable of inducing both humoral and cellular immune response.

The primary endpoint of the MAGRIT study is disease-free survival (DFS) [26, 28].

\section{PRAME}

PRAME (PReferentially Expressed Antigen of MElanoma) was first identified in patients with melanoma, but is also present in patients suffering from NSCLC (both adeno- and squamous cell carcinoma subtypes), breast cancer and kidney cancer, and in patients with acute and chronic leukaemia. The antigen only exhibits expression on a limited number of human tissues: it may be present in the ovaries, endometrium, kidneys and adrenal medulla. Cytotoxic T lymphocytes are capable of lysing cell lines exhibiting PRAME expression, and they do not exhibit activity in relation to non-cancerous cells, except for mature dendritic cells (mDCs) and kidney epithelial cells. The function of the PRAME protein has not yet been fully established, but the data indicates that it is associated with inhibition of the signalling pathway via the retinoic acid receptor (RAR). PRAME protein overexpression has been postulated as a tumour cell mechanism for evading the surveillance of the RAR pathway, which may be associated with poor prognosis. Currently, a phase 2 PEARL study (PRAME as Adjuvant Immunotherapy In Resected non-Small Cell Lung Cancer) is underway, assessing the efficacy of the vaccine. The immunoadjuvant used in this study was AS15 in liposomal form. The study's design is similar to the design of the MAGRIT study. It includes patients radically resected for NSCLC, stages IA (T1b), IB, II or IIIA, whose tumours exhibit PRAME expression. The vaccination begins within 12 weeks in patients not receiving complementary chemotherapy, or within 8 weeks from the last day of chemotherapy. The primary endpoint of the study, similarly to the MAGRIT study, is DFS [29, 30].

\section{Vaccines targeting MUC-1}

MUC-1 is a highly glycosylated transmembrane protein, which exhibits expression on cells of many tumours, including $86 \%$ of adenocarcinomas and nearly $74 \%$ of the remaining NSCLC subtypes. The glycosylation of the extracellular domain of MUC-1 in tumour cells is abnormal, revealing highly immunogenic protein skeletons comprised of repetitive 20-amino-acid sequences [8, 32, 33]. The MUC-1 protein is an anti-adhesion protein engaged in the process of carcinogenesis and tumour cell migration, which causes the tumour cells to be refractory to apoptosis and chemotherapeutic agents. It also plays a role in immunosuppression, inhibiting $\mathrm{T}$ cell proliferation. In patients with NSCLC, both the pattern and the level of MUC-1 expression on tumour cells, as well as the low level of natural antibodies targeting MUC-1, is associated with poor prognosis. Clinical studies assessed two vaccines targeting MUC-1: L-BLP25 and TG4010. 


\section{L-BLP25 (Stimuvax ${ }^{\circledR}$ )}

L-BLP25 is a liposomal vaccine targeting the extracellular core peptide of MUC-1. The vaccine contains an adjuvant monophosphoryl lipid A (MPL) [8, 34], which stimulates the activation of antigen-presenting cells via Toll-like receptors (TLRs), along with 3 other lipids added to ensure better vaccine distribution, and to facilitate its uptake by immune-system cells. Preclinical studies confirm that L-BP25 may induce antigen-specific proliferation of T lymphocytes as well as interferon $\gamma$ secretion [7, 8, 35]. Evidence of immune response being induced by L-BLP25 was observed in 3 early clinical studies encompassing patients who had previously undergone chemotherapy for NSCLC in stage IIIB or IV. The first studies demonstrated that the vaccine may be used safely in doses from 20 to $200 \mu \mathrm{g}$.

A IIB phase trial randomized 171 stage IIIB/IV patients who had achieved a response or stabilisation of the disease after first-line treatment. One arm of the study included patients receiving L-BLP25 and best supportive care (BSC); the patients in the other study arm received only the latter [36]. Patients in the vaccine arm received a single intravenous cyclophosphamide dose of $300 \mathrm{mg} / \mathrm{m}^{2} 3$ days before the first vaccination; it successfully reduced the activity of regulatory $T$ cells. Subsequently, L-BLP25 was administered 8 times in weekly intervals; it was injected into four body regions, in order to increase the distribution of the vaccine molecules among the nearby lymph nodes $[7,37]$. During the $13^{\text {th }}$ week, the patients entered into the supportive phase, which consisted of administering the vaccine every 6 weeks and lasted until disease progression was observed. Median survival time from the date of randomization was longer by 4.4 months in the study arm receiving both L-BLP25 and BSC (17.4 months vs. 13 months). The most significant advantages were observed in patients with locoregional advancement of the disease (stage IIIB), whose median survival time was 30.6 months in the L-BLP25 plus BSC arm, as compared with 13.3 months in the BSC arm [37]. It was established that stage IIIB patients with pleural effusion (according to TNM v. 6.0) and stage IV patients do not benefit in terms of overall survival [38]. Furthermore, it was demonstrated that the vaccine had a good safety profile. The most frequently occurring side effects were mild flu-like symptoms, events related to the administration of cyclophosphamide and mild reactions at the sites of vaccine injections, as well as, non-significant clinically, instances of lymphopaenia. No autoimmune reactions were found [39]. Further research into the efficacy and safety of using L-BLP25 became the aim of large randomized phase III trials: START (Stimulating Targeted Antigenic Response To NSCLC) and INSPIRE (Stimuvax trial In Asian NSCLC Patients: Stimulating Immune Response). The latter was conducted in 5 states (China, Hong Kong, Singapore, South Korea, and Taiwan) and its design is identical to the design of the START study. The START study included stage III patients who achieved an objective response or stabilization of the disease after first-line treatment (chemotherapy based on platinum derivatives + radiation therapy $\geq 50 \mathrm{~Gy}$ ). The patients were randomized into two study arms (vaccine + BSC or BSC alone) in a $2: 1$ ratio. L-BLP25 was well tolerated and no adverse events dependent on the immune system were noted. However, the analysis of the study data did not demonstrate any benefit of using the L-BLP25 vaccine, in terms of extending overall survival. The study encompassed 1513 patients (from January 2007 until November 2011). Median OS was 25.6 months in the L-BLP25 arm vs. 22.3 months in the placebo arm [40]. The reasons behind this failure are ascribed to the lack of exclusion criteria based on biomarker assessment (the exclusion of patients whose tumour tissue material did not exhibit MUC-1 expression), not defining the immunological status of patients (the exclusion of patients with low levels of peripheral T lymphocytes and high levels of immunosuppressive cells, either circulating or located within the tumour), not assessing the immunological response against MUC-1 or other TAAs (the exclusion of patients with poor response), as well as other factors [40]. It is noteworthy that some patient subgroups achieved clear advantages from vaccination. Clinically significant extension of overall survival was observed in the subgroup of patients who had previously undergone combination radiation therapy in comparison with patients after sequential radiation [40]. Designing additional prospective clinical phase III trials is certainly necessary.

\section{TG4010}

TG4010 is a recombinant viral vector (modified Ankara virus), which exhibits expression of the whole sequence of MUC-1 and interleukin-2 (IL-2) - (MVA-MUC-1-IL-2). Interleukin-2 stimulates T-cell response, which is why the vaccine may stimulate or intensify cellular response against tumour cells exhibiting MUC-1 expression [41].

A phase IIB trial assessed the efficacy of TG4010 in previously untreated stage IIIB/IV patients. In the first arm of the study, TG4010 was administered in combination with chemotherapy: cisplatin dosed at $100 \mathrm{mg} / \mathrm{m}^{2}$ on day 1 and vinorelbine dosed at $25 \mathrm{mg} / \mathrm{m}^{2}$ on days 1 and 8 . The patients in the second arm of the study received TG4010 in monotherapy until disease progression; subsequently, they received TG4010 in combination with cytotoxic treatment in the same regimen as in study arm one. In the first study arm, partial response was achieved in 35.1\% of patients, median time until progression was 4.8 months, median overall survival was 12.7 months and one-year survival was achieved by $53 \%$ of patients. In the second study arm, response was achieved in 14\% of patients, median overall survival was 14.9 months and one-year survival was achieved by $60 \%$ of patients. Cellular immune response against MUC-1 was noted in lymphocyte samples of all patients in whom a clinical response was observed. In phase IIB of the trial, stage IIIB/IV patients with previously untreated NSCLC were randomly allocated to the arm receiving only first-line chemotherapy (cisplatin with gemcitabine) or to the arm receiving chemotherapy in combination with TG4010. The vaccine was administered subcutaneously, initially over 6 weeks in weekly intervals, and then every 3 weeks until disease progression. The addition of TG4010 to chemotherapy significantly increased 
the response rate, as well as the percentage of patients achieving 6-month progression-free survival (55\% vs. 35\%). Moreover, a good safety profile of such therapy was established. The most frequent adverse effects consisted of skin reactions at the injection site, fever and abdominal pain. Furthermore, it was proven that adding TG4010 to chemotherapy in the subgroup of patients with normal initial values of activated NK cells (approximately $75 \%$ of patients) leads to significant improvements in terms of overall survival (17.1 vs. 11.3 months) [27, 38, 44].

Normal initial level of activated NK cells is one of the inclusion criteria for the IIB/III phase trial assessing the efficacy of TG4010 combined with first-line chemotherapy in stage IV patients with NSCLC.

\section{EGF vaccine (CIMAvax $\left.{ }^{\circledR}\right)$}

The EGFR (Epidermal Growth Factor Receptor) signalling pathway mediated by EGF ligands is associated with cell proliferation, apoptosis, angiogenesis and metastasis occurrence. EGF overexpression can be observed in many solid tumours and is typically associated with more aggressive disease progression and unfavourable prognosis [45].

The CIMAvax vaccine consists of recombinant human epidermal growth factor (EGF) combined with a Montanide ISA51 immunoadjuvant coupled with recombinant Neisseria meningitidis transport proteins $[46,47]$. A phase II trial included 80 patients with NSCLC (stage IIIB or IV) after the completion of first-line chemotherapy. They were randomly allocated to the arm receiving the EGF vaccine or to the arm receiving $B S C$ in a $1: 1$ ratio $[48,104]$. The first vaccine dose was preceded by cyclophosphamide infusion $\left(200 \mathrm{mg} / \mathrm{m}^{2}\right)$. A single dose of the vaccine contained $50 \mu \mathrm{g}$ of EGF equivalents, and it was administered on days 1,7 , 14, 28 and, subsequently, in monthly intervals. The study demonstrated a decrease in the concentration of EGF in the serum of patients receiving the vaccine, and a strict correlation was found between the antibody titre and the reduction of EGF concentration in the serum. Reduction of EGF concentration to less than $168 \mathrm{pg} / \mathrm{ml}$ was associated with the prolongation of OS (13 months in patients with $168 \mathrm{pg} / \mathrm{ml}$ or less vs. 5.6 months in patients with EGF concentration above $168 \mathrm{pg} / \mathrm{ml}$ ) [49]. High initial EGF concentration is a predictive factor of vaccine response and an adverse prognostic factor for non-vaccinated patients [50]. Although a trend was observed toward prolonging the survival of vaccinated patients, statistical significance was not achieved. However, clinically significant differences in terms of survival length were observed in the subgroup of patients aged 60 years and less in comparison with the control study arm (median survival time: 11.57 months vs. 5.33 months, respectively).

The CIMAvax vaccine is only registered in Cuba, for adult stage IIIB/IV patients with NSCLC. Currently, a clinical phase III trial is underway, which aims to assess the vaccine's efficacy and its influence on overall survival in two patient subgroups: $>60$ years of age and $\leq 60$ years of age. The efficacy and safety of CIMAVax-EGF is also being evaluated by a randomized phase II trial for patients with prostate cancer [50].

\section{Belagenpumatucel-L (Lucanix ${ }^{\circledR}$ )}

Belagenpumatucel- $L$ is the only vaccine produced out of tumour cells that was evaluated by phase III clinical studies. Lucanix is a non-viral, genetically modified, allogenic vaccine obtained from four lung cancer cell lines, including two adenocarcinoma cell lines, one squamous carcinoma cell line and one large-cell carcinoma cell line (H460, H520, SKLU-1, RH2) [50]. The cells are transferred by plasmids containing antisense (transforming growth factor $\beta 2$ (TGF- $\beta 2$ ) transgenes and irradiated [51]. A high level of TGF- $\beta 2$ is usually associated with immunosuppression (disturbance in the activity of NK cells and dendritic cell inhibition), and it may be responsible for unfavourable prognosis in patients suffering from lung cancer [52]. The immunogenicity of the vaccine is heightened by the suppression of TGF- $\beta 2$ production by the tumour, thanks to the expression of antisense RNA in the vaccine plasmid.

The clinical effectiveness and safety of using Belagenpumatucel were first evaluated in a phase II trial, the results of which were published in 2009. The study encompassed 75 stage II-IV patients. They received the vaccine intradermally in one of three doses $\left(1.25,2.5\right.$ or $5 \times 10^{7}$ cells per injection), once every month or once every two months, up to a maximum total of 16 doses. Survival extension was observed in patients in advanced stages of lung cancer who received the dose of $\geq 2.5 \times 10^{7}$ cells/ injection. Estimated 2-year survival rates amounted to $47 \%$ and $18 \%$, respectively. Partial response was achieved by $15 \%$ of patients in the stage IIIB/IV subgroup. Further analysis revealed that patients who developed both cellular and humoral immune response to the vaccination achieved longer OS in comparison with patients without this response. Their median survival time amounted to 32.5 months (95\% Cl: 25.2-39.8 months) in comparison with 11.6 months (95\% Cl: 5.6-17.6 months) [51]. Longer survival in the Belagenpumatucel group was related to the decrease in the amount of circulating tumour cells.

These reports became the foundation for commencing the phase III clinical trial called STOP (Survival, Tumour-free, Overall, Progression-free); its goal was to evaluate the efficacy and safety of using Belagenpumatucel vs. placebo in supportive treatment. The study included stage IIIA (only T3N2), IIIB and IV patients who, after first-line chemotherapy/chemoradiotherapy, achieved total or partial remission or stabilisation of the disease, which lasted for at least a month from the completion of treatment.

Injections were performed 18 times in monthly intervals, with additional vaccination in months 21 and 24 . The primary endpoint of the study was overall survival. The study was terminated early several months ago; no official cause of termination has yet been announced.

\section{Racotumomab (1E10, Vaxira ${ }^{\circledR}$ )}

Racotumomab is a vaccine based on anti-idiotype antibodies, meant to mimic the ganglioside antigens of the tumour. Its design utilises the presence of specific types of sialic acid, including the NGcGM3 ganglioside, on the cells of lung cancer, breast cancer, melanoma and selected types of infantile neoplasms. Of further significance is 
the fact that, while these substances are present on the cells of other mammals, including primates, they are not present on healthy human cells. Cruijsen et al. conducted research on the relation between NGcGM3 expression and angiogenesis. After analysing 176 NSCLC, they found that $90 \%$ of the obtained immunohistochemical reaction results were positive [55]. The vaccine was obtained by immunizing BALB/c mice with P3 antibodies, reacting specifically with NGcGM3. Vaccines containing 1 E10 (racotumomab) are capable of stimulating immunological reaction and producing anti-idiotype antibodies.

In a phase 2 trial, the vaccine was administered to 71 stage IIIB/IV NSCLC patients previously treated with standard radiochemotherapy. The treatment consisted of 5 vaccinations administered every two weeks, followed by 10 injections in 28-day intervals. After the completion of this phase, the patients in good general condition were able to continue their treatment with the same vaccination intervals. The overall survival time from the date of the first vaccination amounted to 9.93 months (8.61-11.25); the one-year survival rate was $34 \%$. The survival time within the group of 56 patients who were in good general condition (ECOG score of PS $=1$ ) and who achieved lasting response to treatment (disease stabilization or partial remission) was 11.5 months [56].

A randomized, multicentre, placebo-controlled double-blinded study included 176 patients with ECOG performance status of 0-2 who had been diagnosed with stage IIIB or IV NSCLC and had completed oncological treatment that resulted in a lasting response in the form of at least disease stabilisation. The patients were allocated in a ratio of $1: 1$ to the experimental arm or to the placebo arm. In both arms of the study, the patients received 5 vaccinations every 2 weeks, followed by vaccinations in 4-week intervals, until resigning from treatment or until their general condition deteriorated. Median overall survival was 8.3 and 6.3 months, and the average for this parameter was 15.7 and 10.6 months, for racotumomab and placebo, respectively $(p=0.02)$. The one-year and two-year survival were 38 and 17 months for the study arm, and 24 and
7 months, respectively, for placebo [57]. Open phase 3 trials are currently underway.

\section{Discussion}

In the field of active immunotherapy in the treatment of NSCLC we observe generally three strategies (Table 1). The first is to add vaccination to first-line chemotherapy. Most trials using this pattern have failed. The only exception is TG 4010 in a population of patients with normal levels of activated NK cells, but due to the retrospective nature of that observation it is currently under prospective validation $[27,38,42]$.

The second way of implementing this treatment in NSCLC is by using it as a tool of "maintenance" in response to first line therapy. Several positive signals from phase II trials were observed, although recently there were negative results from some phase III trials. Further analysis of those results showed some promising findings in two large trials called START and STOP. In the subgroup of patients previously treated with concurrent chemoradiotherapy, results concerning overall survival were positive. Those observations require confirmation in prospective trials, but they seem to be promising. The population of patients who have been treated with chemoradiotherapy in concurrent fashion usually have responses lasting longer, and it probably gives time to develop an immune response.

The last, but not least, strategy is using vaccination in an adjuvant setting. The promising results of a phase II study with MAGE - A3 vaccine prompted a large phase III trial that is currently in its follow-up period, but several years are required for enough events to take place to achieve the possibility of evaluating the immunotherapy effect. A similar situation concerns the PRAME vaccine, but it is currently undergoing a phase II trial.

Although immunotherapy is not yet a standard procedure in the therapeutic management of patients with NSCLC, it may play an important role in the future. An additional advantage offered by such therapy is its low toxicity. Exceptional benefits of employing this treatment can be achieved in selected patient subgroups: it appears that the most significant results are to be expected in the group

Table 1. Clinical trials using therapeutic cancer vaccines in NSCLC

\begin{tabular}{|c|c|c|c|c|}
\hline Vaccine & Adjuvant & Target & Patients & Trial \\
\hline recMAGE-A3 & AS15 & MAGE-A3 & $\begin{array}{c}\text { resectable stage IB-IIIA NSCLC, MAGE-A3 expression } \\
\text { on tumor }\end{array}$ & $\begin{array}{l}\text { MAGRIT/ } \\
\text { NCT00480025 }\end{array}$ \\
\hline PRAME & AS15 & PRAME & $\begin{array}{l}\text { resectable stage IA (T1b)-IIIA NSCLC, } \\
\text { PRAME expression on tumor }\end{array}$ & PEARL \\
\hline $\begin{array}{l}\text { Stimuvax } \\
(\text { L-BLP 25) }\end{array}$ & $\begin{array}{l}\text { monophosphoryl } \\
\quad \text { lipid A }\end{array}$ & MUC1 & $\begin{array}{l}\text { unresectable stage III NSCLC } \\
\text { SD or better after first-line RCT }\end{array}$ & $\begin{array}{l}\text { START/ } \\
\text { NCT00409188 }\end{array}$ \\
\hline TG4010 & viral vector & MUC1 & stage IIIB/IV with first-line chemotherapy & \\
\hline $\begin{array}{l}\text { CimaVax } \\
\text { EGF-Rp64k }\end{array}$ & montanide ISA51 & EGF & $\begin{array}{c}\text { stage IIIB/IV NSCLC } \\
\text { SD or better after first-line chemotherapy }\end{array}$ & NCT00516685 \\
\hline $\begin{array}{l}\text { Lucanix } \\
\text { (Belagenpumatucel-L) }\end{array}$ & $\begin{array}{l}\text { allogenic cell } \\
\text { lines }\end{array}$ & $\begin{array}{c}\text { TGF- } \beta \\
\text { antisense } \\
\text { plasmid }\end{array}$ & $\begin{array}{c}\text { stage IIIA (T3N2)-IV NSCLC, } \\
\text { SD or better after first-line platinum-based CHT }\end{array}$ & $\begin{array}{c}\text { STOP/ } \\
\text { NCT00676507 }\end{array}$ \\
\hline
\end{tabular}


of patients treated adjuvantly or in patients with minimal residual disease. The combination of chemotherapy and immunotherapy in treating advanced diseases also appears promising as it is known that platinum derivatives may induce immunogenic cell death, thus heightening the effect of the vaccine.

The above article focused on the vaccines whose efficacy and safety were evaluated in phase III clinical trials. Although some of the trials ended unsuccessfully, some of the results are promising. Therefore, designing further studies is certainly needed in order to identify the patients for whom immunotherapy may be most advantageous.

The authors declare no conflict of interest.

\section{References}

1. Bradbury PA, Shepherd FA. Immunotherapy for lung cancer. I Thorac Oncol 2008; 3 (6 Suppl 2): S164-70.

2. Coley WB. The treatment of malignant tumors by repeated inoculations of erisipelas. With a report of ten original cases. Clin Orthop Relat Res 1991; (262): 3-11.

3. Coley WB. Late results of the treatment of inoperable sarcoma by mixed toxins of erysipelas and bacillus prodigiosus. Am J Med Sci 1906; 131: 375-430.

4. Carswell EA, Old LJ, Kassel RL, Green S, Fiore N, Williamson B. An endotoxin-induced serum factor that causes necrosis of tumor. Proc Natl Acad Sci U S A 1975; 72: 3666-70.

5. Old LJ, Clarke DA, Benacerraf B. Effect of Bacillus Calmette-Guerin infection on transplanted tumors in the mouse. Nature 1959; 184 (Suppl 5): 291-2.

6. Rosenberg SA, Lotze MT, Muul LM, Leitman S, Chang AE, Etting hausen SE. Observations on the systemic administration of autologous lymphokine-activated killer cells and recombinant interleukin-2 to patients with metastatic cancer. N Engl J Med 1985; 313: 1485-92.

7. Murala S, Alli V, Kreisel D, Gelman AE, Krupnick AS. Current status of immunotherapy for the treatment of lung cancer. J Thorac Dis 2010; 2: 237-44.

8. Mellstedt H, Vansteenkiste J, Thatcher N. Vaccines for the treatment of non-small cell lung cancer: Investigational approaches and clinical experience. Lung Cancer 2011; 73: 11-7.

9. Upadhyaya SK. Immunotherapy for cancer with special reference to lung cancer. Apollo Medicine 2006; 3: 277-84.

10. Igney FH, Krammer PH. Immune escape of tumors: apoptosis resistance and tumor counterattack. J Leukoc Biol 2002; 71: 907-20.

11. Whiteside TL. Immune suppression in cancer: effects on immune cells, mechanisms and future therapeutic intervention. Semin Cancer Biol 2006; 16: 3-15.

12. Widen K, Mozaffari F, Choudhury A, Melstedt H. Overcoming immunosuppressive mechanisms. Ann Oncol 2008; 19: 241-7.

13. Korst RJ, Crystal RG. Active, specific immunotherapy for lung cancer: hurdles and strategies using genetic modification. Ann Thorac Surg 2003; 76: 1319-26.

14. Toes R, Offringa R, Blom R, Melief CJ, Kast WM. Peptide vaccination can lead to enhance tumor growth through specific $T$ cell tolerance induction. Proc Natl Acad Sci U S A 1996; 93: 7855-60.

15. Akhurst RJ, Derynck R. TGF-beta signaling in cancer - a double-edged sword. Trends Cell Biol 2001; 11: S44-51.

16. Mocellin S, Wang E, Marincola FM. Cytokines and immune response in the tumor microenvironment. J Immunother 2001; 24: 392-407.
17. Korkolopoulou P, Kaklamanis L, Pezzella F, Harris AL, Gatter KC. Loss of antigen-presenting molecules (MHC class I and TAP-1) in lung cancer. Br J Cancer 1996; 73: 148-153.

18. Walker PR, Saas P, Dietrich PY. Role of Fas ligand (CD95L) in immune escape: the tumor cell strikes back. J Immunol 1997; 158: 4521-4.

19. Sienel W, Varwerk C, Linder A, Kaiser D, Teschner M, Delire. M Melanoma-associated antigen (MAGE-A3) expression in stages I and II non-small cell lung cancer; results of a multi-center study. Eur J Cardiothorac Surg 2004; 25: 131-4.

20. Brichard VG, Ejeune D. GK's antigen-specyfic cancer immunotherapy programme: pilot results leading to phase III clinical development. Vaccine 2007; 25: B61-71.

21. Grah J, Samija M, Juretić A, Sarcević B, Sobat H. Immunohystochemical expression of cancer/testis antigens (MAGE-A3/4, NY-ESO-1) in non-small cell lung cancer: the relationship witch clinical-pathological features. Coll Antropol 2008; 32: 731-6.

22. Vansteenkiste JF, Zielinski M, Dahabreh IJ, Linder A. Association of gene expression signature and clinical efficacy of MAGE-A3 antigen-specific cancer immunotherapeutic (ASCl) as adjuvant therapy in resected stage IB/II non-small cell lung cancer. J Clin Oncol 2008; 26 (15S Suppl.): 7501.

23. Tyagi P, Mirakhur B. MAGRIT: the largest-ever phase III lung cancer trial aims to establish a novel tumor-specific approach to therapy. Clin Lung Cancer 2009; 10: 371-4.

24. Schultz ES, Lethe B, Cambiaso CL, et al. A MAGE-A3 peptide presented by HLA-DP4 is recognized on tumor cells by CD4+ cytolytic T Lymphocytes. Cancer Res 2000, 60: 6272-5.

25. Winter $\mathrm{H}$, van den Engel NK, Rusan M, et al. Active-specific immunotherapy for non-small cell lung cancer. J Thorac Dis 2011; 3 (2): 105-14.

26. Vansteenkise J, Zieliński $M$, Linder $A$, et al. Final results of a multi-center, doube-blind, randomized, placebo-controlled phase II study to assess the efficacy of MAGE-A3 immunotherapeutic as adjuvant therapy in stage IB/II nn-small cell lung cancer (NSCLC). J Clin Oncol 2007; 25: abstract 7554.

27. Brahmer JR. Harnessing the immune system for the treatment of non-small cell lung cancer. J Clin Oncol 2013; 31: 1021-8.

28. Shepherd FA, Douillard JY, Blumenschein GR. Immunotherapy for Non-small cell lung cancer. Novel Approaches to improve patient outcome. J Thorac Oncol 2011; 6: 1763-73.

29. Roman-Gomez J, Jimenez-Veasco A, Agirre X. Epigenetic regulation of PRAME gene in chronic myelotic leukaemia. Leuk Res 2007; 31: 1521-8.

30. Epping MT, Wang L, Edel MJ. The human tumor antigen PRAMEis a dominant repressor of retinoic acid receptor signalling. Cell 2005; 122: 835-47.

31. Vansteenkiste J, Zielinski M, Dahabreh IJ, et al. Association of gene expression signature and clinical efficacy of MAGE-A3antigen-specific cancer immunotherapeutic (ASCl) as adjuvant therapy in resected stage IB/II non-small cell lung cancer (NSCLC). J Clin Oncol 2008; 26 (15 Suppl.): 397s.

32. Grinstead JS, Koganty RR, Krantz MJ, Longenecker BM, Campbell AP. Effect of glycosylation on MUC1 humoral immune recognition: NMR studies of MUC1 glycopeptide-antibody interactions. Biochemistry 2002; 17: 391-9.

33. Agrawal B, Longenecker BM. MUC1 mucin-mediated regulation of human T cells. Int Immunol 2005; 17: 391-9.

34. Guddo F, Giatromanoaki A, Koukourakis MI, et al. MUC1 (episialin) expression in non-small cell lung cancer is independent of EGFR and c-erbB-2 expression and correlates with poor survival in node positive patients. J Clin Pathol 1998; 51: 667-71.

35. Woenckhaus M1, Merk J, Stoehr R, et al. Prognostic value of FHIT, CTNNB1 and MUC1 expression in non-small cell lung cancer. Hum Patol 2008; 39: 126-36.

36. Hirasawa Y, Kohno N, Yokoyama A, Kondo K, Hiwada K, Miyake M. Natural autoantibody to MUC1 is a prognostic indicator for nonsmall cell lung cancer. Am J Respir Crit Care Med 2000; 161: 589-94.

37. Butts C, Maksymiuk A, Goss G, et al. A multi-centre phase IIB randomized controlled study of BLP25 liposome vaccine (L-BLP25 or Stimuvax) for active specific immunotherapy of non-small cell lung cancer (NSCLC): updated survival analysis. J Thorac Oncol 2007; 2: s332-3. 
38. Decoster L, Wauters I, Vansteenkiste JF. Vaccination therapy for non-small cell lung cancer: review of agents in phase III development. Ann Oncol 2012; 23: 1387-93.

39. Butts C, Anderson H, Maksymiuk A, et al. Long-term safety of BLP25 liposome vaccine (L-BLP25) in patients with stage IIIB/IV non-small cell lung cancer (NSCLC). J Clin Oncol 2009; 27(15 Suppl.): abstract 3055 .

40. Butts C, Socinski MA, Mitchell P. START: A phase III study of L-BLP25 cancer immunotherapy for unresectable stage III non-small cell lung cancer. J Clin Oncol 2013; 31 (suppl): abstract 7500.

41. Kroemer G, Zitvogel L, Galluzzi L. Victories and deceptions in tu mor immunology. Oncoimmunology 2013; 2: e23687

42. Ramlau R, Quoix E, Rolski J, et al. A phase II study of TG4010 (MVA MUC1-IL-2) In association with chemotherapy In patients with stage III/IV non-small cell lung cancer. J Thorac Oncol 2008; 3: 735-44.

43. Acres B, Quoix E, Ramlau R, et al. Biomarkers associated with clinical outcome in advanced non-small cell lung cancer patients treated with TG4010. J Clin Oncol 2009; 27 (15 Suppl.): abstract 3027.

44. Ramlau R, Weestel V, Papai Z, et al. Randomized phase IIB trial evaluating the therapeutic vaccine TG4010 (MVA-MUC1-IL-2) as an adjunct to chemotherapy in patients with advanced non-small cell lung cancer (NSCLC). J Clin Oncol 2008; 26 (15S Suppl.): abstract 8023.

45. Limacher J.M, Quoix E. TG4010: A therapeutic vaccine against MUC1 expressing tumors. Oncoimmunology 2012; 1: 791-2.

46. Reissmann PT, Koga H, Figlin RA, Holmes EC, Slamon DJ. Amplification and overexpression of the cyclin D1 and epidermal growth factor receptor genes in non-small-cell lung cancer. Lung Cancer Study Group. J Cancer Res Clin Oncol 1999; 125: 61-70.

47. González G, Crombet T, Catalá M, Mirabal V, Hernández JC, González Y, Marinello P, Guillén G, Lage A. A novel cancer vaccine composed of human-recombinant epidermal growth factor linked to a carrier protein: report of a pilot clinical trial. Ann Oncol 1998; 9: 431-435.

48. Rodríguez PC, Rodríguez G, González G, Lage A. Clinical development and perspectives of CIMAvax EGF, Cuban vaccine for nonsmall cell lung cancer therapy. MEDICC Rev 2010; 12: 17-23.

49. Neninger Vinageras E, de la Torre A, Osorio Rodríguez M, et al. Phase II randomized controlled trial of an epidermal growth factor vaccine in advanced non-small cell lung cancer. J Clin Oncol 2008; 26: 1452-1458.

50. Crombet Ramos T, Neninger E, Gonzales J. EGF-based cancer vaccine: Optimizing predictive and surrogate biomarkers. J Clin Oncol 2013; 31 (suppl): abstract 3013.

51. Gardia B, Neninger E, de la Torre A et al. Effective inhibition of the epidermal growth factor/epidermal growth factor receptor binding by anti-epidermal growth factor antibodies is related to better survival in advanced non-small-cell lung cancer patients treated with the epidermal growth factor cancer vaccine. Clin Cancer Res 2008; 14: 840-6.

52. Nemunaitis J, Dillman RO, Schwarzenberger PO, et al. Phase II study of belagenpumatucel-L, a transforming growth factor beta-2 antisense gene-modified allogenic tumor cell vaccine in non-small cell lung cancer. J Clin Oncol 2006; 24: 4721-30.

53. Fakhrai H, Tong A, Nemunaitis J, et al. Correlation of immune responses and survival in a phase II study of belagenpumatucel- $L$ in non-small cell lung cancer. J Clin Oncol 2009; 27 (15S).

54. Kong F, Jirtle RL, Huang DH, Clough RW, Anscher MS. Plasma transforming growth factor-beta 1 level before radiotherapy correlates with long term outcome of patients with lung carcinoma. Cancer 1999; 86: 1712-9.

55. van Cruijsen H, Ruiz MG, van der Valk P, de Gruijl TD, Giaccone G. Tissue micro array analysis of ganglioside Nglycolyl GM3 expression and signal transducer andactivator of transcription (STAT-3) activation in relation to dendritic cell infiltration and microvessel density in non-small cell lung cancer. BMC Cancer 2009; 9: 180.

56. Alfonso S, Diaz RM, de la Torre A, et al. 1 E10 anti-idiotype vaccine in non-small cell lung cancer. Experience in stage IIIB/IV patients. Cancer Biol Ther 2007; 6: 1847-52.
57. Macias A, Alfonso S, Santiesteban E. Active specific immunotherapy with racotumomab in the treatment of advanced non-small cell lung cancer. Ann Oncol 2012; 23 suppl 9: abstract 1238PD.

\section{Address for correspondence}

\section{Katarzyna Szyszka-Barth}

Wielkopolskie Centrum Pulmonologii i Torakochirurgii

Szamarzewskiego 62

60-569 Poznan, Poland

tel. +4861665 4290

e-mail: katarzyna.barth@o2.pl

Submitted: 4.03.2014

Accepted: 17.04 .2014 